\title{
Critique génétique et philologie : racine de la différence
}

Daniel Ferrer

\section{(2) OpenEdition \\ 1 Journals}

\section{Édition électronique}

URL : https://journals.openedition.org/genesis/98

DOI : 10.4000/genesis.98

ISSN : 2268-1590

\section{Éditeur :}

Presses universitaires de Paris Sorbonne (PUPS), Société internationale de génétique artistique littéraire et scientifique (SIGALES)

\section{Édition imprimée}

Date de publication : 20 juin 2010

Pagination : 21-23

ISBN : 978-2-84050-697-3

ISSN : 1167-5101

\section{Référence électronique}

Daniel Ferrer, «Critique génétique et philologie : racine de la différence », Genesis [En ligne], 30 | 2010, mis en ligne le 30 mai 2012, consulté le 30 mars 2023. URL : http://journals.openedition.org/genesis/ 98 ; DOl : https://doi.org/10.4000/genesis.98 


\section{Critique génétique et philologie : racine de la différence}

Daniel Ferrer

Le travail du généticien penché sur ses brouillons peut paraître semblable à celui du philologue qui se sert des manuscrits pour établir un texte. Mais les deux perspectives n'en sont pas moins fondamentalement différentes - ce qui ne signifie pas qu'elles soient mutuellement exclusives. On peut mettre bien des choses sous l'appellation de philologie : on entendra ici par ce mot la « critique textuelle», cette pratique séculaire, voire millénaire, qui consiste à critiquer un texte, à l'examiner pour s'assurer de son authenticité et de sa pureté, à des fins religieuses ou culturelles.

Les liens de filiation entre les deux disciplines ont été rappelés et les circonstances qui ont entouré leur naissance respective ont été analysées avec finesse ${ }^{1}$; mais il faut, au moins une fois, rendre compte de leur différence en des termes qui ne sont pas génétiques, ni même historiques. Pour dire les choses en deux mots et pour simplifier à l'extrême, la philologie s'intéresse à la répétition du texte, tandis que la critique génétique s'intéresse au processus de création, c'est-à-dire à l'invention; l'une vise à établir le texte en le faisant émerger de la foule de ses incarnations accidentelles, alors que l'autre a plutôt pour effet de le déstabiliser en le confrontant à l'ensemble de ses brouillons.

La critique textuelle, aussi dite critique de restitution, a pour but le retour en arrière, la remontée vers l'origine. Elle bute sur une impossibilité qui est en même temps sa raison d'être : l'origine est toujours perdue, pervertie par les corruptions, il est donc impossible de la répéter simplement. Plus grave encore, il apparaît que la corruption est inhérente à la reproduction du texte.

La philologie considère, à juste raison, que toute copie est défectueuse. Chaque duplication introduit de nouvelles erreurs. La répétition met en échec la répétition. Il suffit de jouer au « téléphone arabe » pour s'en persuader.

À partir de cette constatation (l'inéluctabilité de l'erreur), la critique textuelle va se pencher avec la plus grande attention sur ces échecs de la répétition qui font obstacle à la remontée vers les origines, et mettre au point une véritable science de l'erreur, de plus en plus sophistiquée. Cet intérêt pour l'erreur devient une véritable fascination, au point que certains philologues finissent par parler des textes comme de simples « supports de faute », un peu comme la psychanalyse autre discipline fondée sur l'étude de la répétition - tend à considérer le texte comme un simple réservoir de lapsus et symptômes divers. Le rapprochement entre ces deux disciplines est d'ailleurs moins saugrenu qu'il n'y parait : Sebastiano Timpanaro a proposé une critique très serrée de la Psychopathologie de la vie quotidienne sur les bases de la philologie lachmannienne et la psychanalyse, de son côté, aurait beau jeu de proposer une analyse des fantasmes véhiculés par les manuels de philologie...

La critique textuelle s'exhorte régulièrement à cesser de parler d'erreurs pour parler d'innovations; et à considérer que l'histoire de la tradition présente autant d'intérêt que la reconstitution des origines... Mais quand bien même cette révolution culturelle serait accomplie, il resterait la distinction entre une innovation qui s'ignore et survient comme interférence dans un processus de transmission, et une innovation qui se revendique comme telle (à tort ou à raison). Le travail d'un copiste saoul qui, à partir de l'annuaire téléphonique, aboutirait à un texte aussi innovant que Finnegans Wake relèverait sans doute de la philologie - celui de Pierre Ménard réécrivant Don Quichotte à l'identique relèverait en revanche certainement de la critique génétique.

1. Voir Bernard Cerquiglini (Éloge de la variante. Histoire critique de la philologie, Paris, Éditions du Seuil, coll. « Des travaux », 1989), JeanLouis Lebrave ( La critique génétique : une nouvelle discipline ou un avatar moderne de la philologie », Genesis, n 1, 1992, p. 33-72) et l'ensemble des travaux de Michel Espagne et Michael Werner sur ces questions. 
Si l'erreur peut être objet d'une science, c'est bien sûr dans la mesure où elle ne reste pas hapax irréductible et s'inscrit dans un processus de répétition - mais la philologie va plus loin et découvre que la répétition est au principe même de l'erreur, à tous les niveaux d'analyse : dittographies (répétition parasite des lettres), " saut du même au même » (répétitions internes au texte qui viennent court-circuiter la répétition $d u$ texte), lectio facilior (répétition paresseuse d'une norme)... Surtout elle découvre ou postule que les erreurs perdurent en persistant de copie en copie (répétition des échecs de la répétition), ce qui permet d'établir la filiation des exemplaires et de tracer le chemin d'une remontée à la source (paradoxe qui a frappé les philologues eux-mêmes : seules les «mauvaises leçons » permettent de définir les bonnes). La critique textuelle va donc entreprendre de mettre en œuvre un jeu complexe entre répétitions corruptrices et répétitions salutaires, faisant jouer l'un contre l'autre des principes apparemment contradictoires (unicité de la leçon authentique vs eliminatio lectionum singularium, lectio difficilior vs usus scribendi), mais qui concourent tous à la plus grande gloire d'une répétition d'ordre supérieur.

Le fondement de toute cette subtile dialectique est un phénomène banal, dont on ne parle jamais tant il paraît évident. Il faut pourtant l'expliciter pour bien saisir la différence entre la philologie et la critique génétique. Il s'agit de l'ordre implicite de répétition qui accompagne chaque texte. On peut dire que chaque texte comporte, quelle que soit la signification qu'il véhicule, une dimension prescriptive implicite qui enjoint la répétition, et plus précisément l'exactitude de la répétition. Un peu comme une partition musicale véhicule une suite d'instructions prescrivant la manière dont un morceau doit être exécuté, le texte transmet lui aussi une instruction, un unique commandement : répétez-moi sans rien changer. Comme une œuvre musicale qui ne prend corps qu'à partir du moment où les prescriptions de la partition sont exécutées, le texte n'existe, ou du moins ne subsiste, qu'à la condition que soit respecté cet ordre de répétition.

Il arrive que cet ordre prenne une forme explicite : c'est l'imprimatur de l'Église, ou encore l'« autorisation de reproduction » de la thèse ; dans la pratique éditoriale moderne, c'est le rôle joué par le "bon à tirer » et la signature qui le valide. Cette injonction explicite revêt parfois une forme hypertrophiée : ainsi la batterie de contrats notariés, de testaments et de codicilles qu'avait élaborés Chateaubriand pour s'assurer de la transmission fidèle des Mémoires d'outre-tombe, ou bien les nombreux testaments dont Stendhal accompagne les manuscrits de Lucien Leuwen et de la Vie de Henry Brulard pour en prescrire les modalités de publication. On peut citer encore la suscription que Rousseau ajouta à ses Dialogues, avant d'aller les déposer sur l'autel de Notre-Dame, demandant à la Providence qu'elle fasse « tomber [son texte] en des mains jeunes et fidèles, qui le transmettent exempt de fraude à une meilleure génération »; ou les menaces de Chaucer adressées à son scribe sous la forme d'un poème (Unto Adam His Own Scriveyne) lui enjoignant la fidélité de copie sous peine de malédiction. Mais signatures, actes juridiques et imprécations comminatoires ne sont que des concrétisations redondantes de l'acte de langage analogue performé en silence par tout texte.

Cet impératif se laisse peut-être plus facilement saisir sous sa forme négative : on songe là aussi à Rousseau et au véritable bon à tirer négatif que constitue la Déclaration relative à différentes réimpressions de ses ouvrages, désavouant «tous les livres anciens ou nouveaux, qu'on imprime et imprimera désormais sous son nom »; à la lettre de Kafka demandant qu'on brûle ses manuscrits ou au testament de Saint-John Perse enjoignant de détruire « tout ce qui pourra être trouvé de moi »; mais aussi plus simplement au deleatur des épreuves d'imprimerie, ou même à la banale rature. Le trait rayant une lettre, un mot, une phrase ou un paragraphe constitue bien une injonction de ne pas recopier ce qui est barré. Quiconque a essayé de faire imprimer un mot sous rature, comme c'était la mode dans les années soixante-dix, sait de quelles précautions il faut s'entourer, de quelles adjonctions étagées de métalangage il faut accompagner son manuscrit pour annuler l'annulation du texte que constitue la rature pour l'imprimeur, ou plus exactement pour annuler l'annulation explicitée par la rature de l'ordre implicite de répéter... Quand on édite des brouillons, et donc qu'on reproduit des ratures, on fait surgir un conflit entre deux injonctions opposées, deux ordres antagonistes de répétition.

Ce n'est pas un hasard si la rature, par opposition aux diverses formes d'effacement et de grattage, fait pour l'essentiel son apparition dans les textes du notariat italien 
du XIII ${ }^{e}$ siècle et plus précisément dans les brouillons, conservés pour mémoire, des actes juridiques. Elle correspond en effet à une dissociation de la valeur légale, performative (annulée) et du contenu informationnel (qui subsiste sous barrure, pour mémoire). On peut également se référer à la pratique d'écrivains tels que Flaubert ou Joyce qui ont l'habitude de rayer leurs brouillons une fois qu'ils en ont intégré le contenu à l'étape suivante de leur rédaction : cette barrure ne représente nullement un désaveu, mais une annulation explicite de l'ordre implicite de répétition (puisque cet ordre a déjà été exécuté).

C'est que le brouillon est un objet plus complexe que le texte. Pour simplifier et en considérant à l'échelle du document ce qui devrait être analysé à l'échelle de la phrase, voire du trait de plume, et en cristallisant en deux phases distinctes ce qu'il faudrait penser comme une pulsation continue, on peut dire que cet objet a deux versants. Sur l'un d'eux, il peut être considéré comme un texte, fût-il provisoire, porteur de l'exigence de répétition. C'est le brouillon en tant qu'il est destiné à être recopié par l'imprimeur, par un copiste ou par l'auteur lui-même. Mais sur son autre versant, le brouillon est au contraire espace d'invention. Et invention s'oppose ici à répétition - même si l'invention ne consiste parfois que dans le montage d'éléments antérieurs ; même si l'invention ne peut être recevable (y compris pour l'auteur lui-même) sans être immédiatement intégrée dans une chaîne de répétitions ; et même si le projet d'une science de l'invention écrite (que la critique génétique se propose d'être) suppose la recherche de régularités qui ne peuvent qu'émousser la pointe de l'originalité.

L'espace de l'innovation que dessinent les brouillons d'écrivains est un espace ouvert, parce qu'il n'est pas circonscrit par le « répétez », commandement unique qui circonscrit l'espace textuel. Il est au contraire traversé par un extraordinaire foisonnement d'injonctions diverses qu'il appartient au généticien d'interpréter. C'est là l'essentiel de son entreprise - l'établissement philologique de la littéralité des brouillons restant pour lui accessoire, ou du moins instrumental, de même que les considérations génétiques demeurent, en principe, une retombée marginale de l'entreprise philologique.

Daniel Ferrer est codirecteur de la rédaction de Genesis.

Daniel Ferrer, daniel.ferrer@ens.fr 